\title{
Compact picosecond mid-IR PPLN OPO in burst-mode operation
}

\author{
Yudi Wu, Sijing Liang, Qiang Fu, Lin Xu, David J. Richardson \\ Optoelectronics Research Centre, University of Southampton, Southampton, SO17 1BJ, UK
}

Short-pulsed mid-infrared (MIR) lasers have many important applications including spectroscopy, material processing and free space communication, where pulse parameters such as a high repetition rate and high peak power can play a critical role in enhancing features e.g. processing speed and signal-to-noise ratio [1]. Optical parametric oscillators (OPOs) are commonly used as MIR laser sources but they require temporal synchronisation between the pump and signal when used to produce short pulses [2]. Hence, picosecond (ps) OPO systems normally operate with a short cavity length for high repetition rate pulses with low peak power, or with a relatively long cavity length for pulses at low repetition rate and high peak power. Here we report a compact short-cavitylength ps OPO that can still provide high-peak-power pulses through burst-mode operation. MIR pulses at a high repetition rate of $1.5 \mathrm{GHz}$ and controllable peak powers up to a maximum of $1.2 \mathrm{~kW}$ are demonstrated.

The pump source for the OPO is a 1040-nm gain-switched laser-diode (GSLD) seeded Ytterbium fibre Master Oscillator Power Amplifier (MOPA) system, similar to the MOPA described in reference [3]. The GSLD technique offers highly controllable pulses at a choice of repetition rates and with flexible pulse duration. In this work, a pulse width of $37 \mathrm{ps}$ and repetition rate of $1.5 \mathrm{GHz}$ are selected for the experiment. In the MOPA, an electro-optic modulator (EOM) is employed as a time gate to suppress a variable number of pulses every $1 \mu$ s to enable burstmode operation with a 1-MHz burst repetition rate (Fig. 1a). The MOPA generates 14-W of maximum average output power for all numbers of pulses-per-burst.

The OPO is of a signal-resonant linear cavity design consisting of two concave mirrors with a 40-mm-long periodically poled lithium niobate (PPLN) nonlinear gain medium. Both mirrors are of high reflectivity at a wavelength of $1.5 \mu \mathrm{m}$ (signal) and $\sim 81 \%$ transmission at both $1 \mu \mathrm{m}$ (pump) and $3 \mu \mathrm{m}$ (idler). The cavity length was set at just $15 \mathrm{~cm}$ to allow synchronous pumping every two signal roundtrips at a $1.5 \mathrm{GHz}$ repetition rate. The PPLN crystal, having 5 channels of grating periods ranging from $29.52-31.59 \mu \mathrm{m}$, is mounted in an oven that allows temperature tuning from 20 to $200{ }^{\circ} \mathrm{C}$, from which an idler wavelength tunability of $2260-3573 \mathrm{~nm}$ was realized (Fig. 1b).
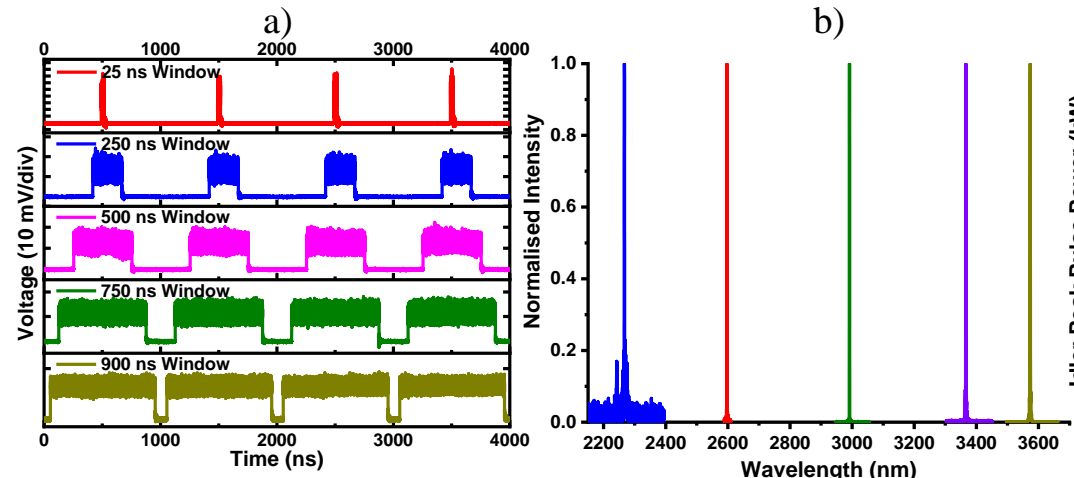

b)

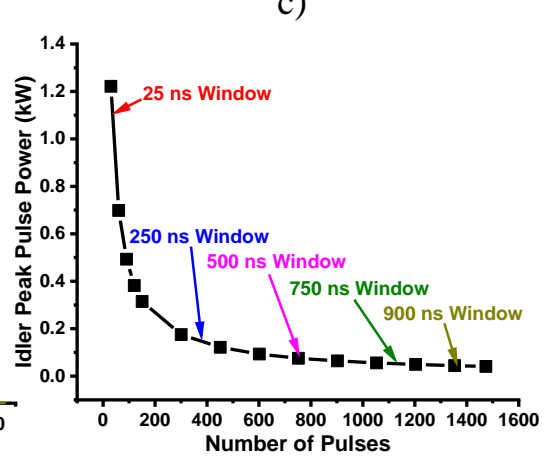

Fig. 1 a) Burst-pulse traces of pump with different time window, b) tunable spectrum of idler, c) idler single-pulse peak power against the number of pulses per burst.

By varying the burst-pulse window with the MOPA's EOM, different numbers of pulses in each burst were generated from the OPO. The average power of the idler output was measured to vary from 1.4 to $2.4 \mathrm{~W}$, dependent upon the window setting. The corresponding peak power of each individual idler pulse of was then calculated and Fig. 1c shows the pulse peak power against the number of pulses for each burst mode. As the number of pulses per burst decreases, the idler pulse peak power increases significantly. With 20 pulses per burst, an idler pulse peak power of $1.2 \mathrm{~kW}$ was achieved. This is two orders of magnitude higher than the idler pulse peak power of $\sim 30 \mathrm{~W}$ achieved when the OPO was operating with a continuous $1.5 \mathrm{GHz}$ train of pulses. Thus, by using burstmode operation, a highly compact MIR OPO with high intra-burst repetition rate as well as high peak power was achieved.

\section{References}

[1] Y. Su, W. Wang, X. Hu, H. Hu, X. Huang, Y. Wang, J. Si, X. Xie, B. Han, H. Feng, Q. Hao, G. Zhu, T. Duan, and W. Zhao, "10 Gbps DPSK transmission over free-space link in the mid-infrared," Opt. Express 26, 34515 (2018)

[2] Q. Fu, L. Xu, S. Liang, P. C. Shardlow, D. P. Shardlow, S. Alam, and D. J. Richardson, "High-average-power picosecond mid-infrared OP-GaAs OPO," Opt. Express 28, 5741 (2020)

[3] H. Chan, S. Alam, L. Xu, J. Bateman, D. J. Richardson, and D. P. Shepherd, "Compact, high-pulse-energy, high-power, picosecond master oscillator power amplifier," Opt. Express 22, 21938 (2014) 